\title{
Effect of Lavender on Sleep, Sexual Desire, Vasomotor, Psychological and Physical Symptom among Menopausal and Elderly Women: A Systematic Review
}

\author{
Nasibeh Roozbeh ${ }^{1}$, Masumeh Ghazanfarpour ${ }^{2}$, Talat Khadivzadeh ${ }^{3}$, Leila Kargarfard ${ }^{4}$, Fatemeh Rajab Dizavandi ${ }^{5}$, \\ Khatereh Shariati ${ }^{6}$ \\ ${ }^{1}$ Mother and Child Welfare Research Center, Hormozgan University of Medical Science, Bander Abbas, Iran, ${ }^{2}$ Department of Midwifery, \\ Razi School of Nursing and Midwifery, Kerman University of Medical Sciences, Kerman, Iran, ${ }^{3}$ Evidence-Based Care Research Centre, \\ Department of Midwifery, School of Nursing and Midwifery, Mashhad University of Medical Sciences, Mashhad, Iran, ${ }^{4}$ Department of \\ Fatemeh, School of Nursing and Midwifery, Shiraz University of Medical Science, Shiraz, Iran, ${ }^{5}$ Department of Community Health and \\ Psychiatric Nursing, School of Nursing and Midwifery, Mashhad University of Medical Sciences, Mashhad, Iran, ${ }^{6}$ Department of Medical \\ Education, Mashhad University of Medical Sciences, Mashhad, Iran
}

Objectives: The present systematic review was conducted to compare the effect of lavender on the quality of sleep, sexual desire, and vasomotor, psychological and physical symptoms among menopausal and elderly women.

Methods: There were five electronic databases which selected to search respective articles which included were Scopus, PubMed, Web of Science, Google Scholar, and the Cochrane Library without any language restriction since the study inception to March 10, 2018. The quality of studies was assessed in accordance with a jaded scale.

Results: According to three trials, the lavender as utilized in a capsule form or aromatherapy could significantly improve the measured Pittsburgh Sleep Quality Index score $(P<0.05)$ in the menopausal and elderly women. The aromatherapy with lavender improved sexual function $(P<0.001)$, depression $(P<0.001)$, anxiety $(P<0.001)$, and physical $(P<0.001)$ symptoms. Based on a trial, 66.7\%, $70.0 \%$, and $53.3 \%$ of subjects reported feelings of relaxation, happiness, and cleanness effects of having used lavender respectively.

Conclusions: The results suggested the effectiveness of the use of lavender either in capsule form or aromatherapy on the improved quality of sleep, depression, anxiety, sexual desire, and psychological and physical symptoms. These results, however, should be interpreted with caution considering the limitations of the study.

Key Words: Hot flashes, Lavandula, Libido, Menopause, Systematic review

\section{INTRODUCTION}

Menopause is a natural life cycle of all females [1] that occurs following the estrogen deficiency, probably resulting in insomnia, irritability, and depression [2]. Reportedly, the menopausal symptoms ranging from mild to severe are found in $65 \%$ to $68 \%$ of females [3], among which hot flashing is the most frequent complaint stated [4].
Over one-third of the life of females is spent after menopause. Hence, the welfare and health needs of menopausal females must be meet to prevent socieconomical harms and improve the life expectancy and the stability of menopause age $[5,6]$.

The vasomotor symptoms and other menopauserelated complications can be attenuated by hormone therapy (HT). However, HT was associated with some side effect such breast cancer, coronary heart disease

Received: April 4, 2019 Revised: July 12, 2019 Accepted: July 28, 2019

Address for Correspondence: Masumeh Ghazanfarpour, Department of Midwifery, Razi School of Nursing and Midwifery, Kerman University of Medical Sciences, Haft-Bagh Highway, Kerman 7616913555, Iran

Tel: 98-91-3633-0656, E-mail: Masumeh.Ghazanfarpour@yahoo.com, ORCID: https://orcid.org/0000-0003-4639-3711 
and pulmonary embolism [2,7]. Therefore, non-hormonal substances, like phytoestrogens, as a safe remedy have been attracted further attentions of postmenopausal women [8]. Approximately, $80 \%$ of peri- and postmenopausal females have been consuming dietary supplements, of which $60 \%$ to $70 \%$ reported these beneficial for health. Most women believe that these natural and safe herbal products as alternatives to traditional medicine are in line with their values and lifestyle [5]. Lavender, Lavandula angustiifolia, because of having linalool, linalyl acetate, ocimenem carypphyllene oxide, local anesthetic tannins, coumarin, flavonoid, and phytosterol [9] have several positive and active effects, such as sedative [10], antidepressant [11], and antioxidant [12] properties, without any determined side effects, except for vomiting and skin dermatitis in topical form [1]. The female life can be affected by adverse effects of menopausal symptoms, highlighting the importance of appropriate treatment of these symptoms. Regarding the menopausal and elderly women tendency towards herbal supplementation and no review on the effect of lavender on menopausal symptoms, a comprehensive systematic review is required to investigate the efficacy of this supplement. Accordingly, the current systematic review was designed to assess the effectiveness of lavender in either capsule form or aromatherapy lavender on quality of sleep, sexual desire, psychological and physical symptom among menopausal and elderly women.

\section{MATERIALS AND METHODS}

\section{Search strategy}

The five electronic databases selected to search the relevant articles were Scopus, PubMed, Web of Science, Google Scholar, and Cochrane Library without any language restriction since inception to March 10, 2018. Inclusion criteria in this systematic review were all clinical trials with control group assessing the efficacy of lavender on the menopausal or elderly women.

The articles, regardless of the type of active ingredients used for aromatherapy, the method of using aromatherapy (massage and inhalation) and the type of treatment used for the control group were enrolled in the study.

The measurement outcomes were at least one of the symptoms including anxiety, depression, and hot flash, psychological, physical and sexual functions. The study design was pre/post trials with or without control group. Search keywords was (lavender OR Lavandula)
AND (menopause OR elderly).

\section{Quality assessment}

The quality of studies was evaluated in accordance with the Jaded scale consisting of randomization (mentioned randomization, appropriate or inappropriate randomization), blinding (mention blinding, appropriate or inappropriate blinding) and account of all patient [13]. The quality of studies was examined independently by two authors. Third party resolved any disagreement between assessors. Other two important items included by research team to jaded scale were intention to treat and baseline comparability.

\section{Data extraction}

Data were collected independently by two authors using a research-made checklist consisting of demographic characteristics such as name of authors, year of publication, age of participants, location of study, type of study, type of treatment and dosage, duration of treatment, percentage of drop out, assessment tool used, number of subject, intervention and control groups, and summary of result.

\section{RESULTS}

Figure 1 showed the process of selection of studies included into systematic review. Table 1 showed characteristics of 4 randomized placebo-controlled trials included to systematic. Table 2 showed the risk of bias assessment using Cochrane Collaboration's tool.

\section{Vasomotor symptoms}

Nikjou et al. [14] conducted a double-blinded crossover clinical trial. The menopausal women with complaint of hot flash were randomized into two equal

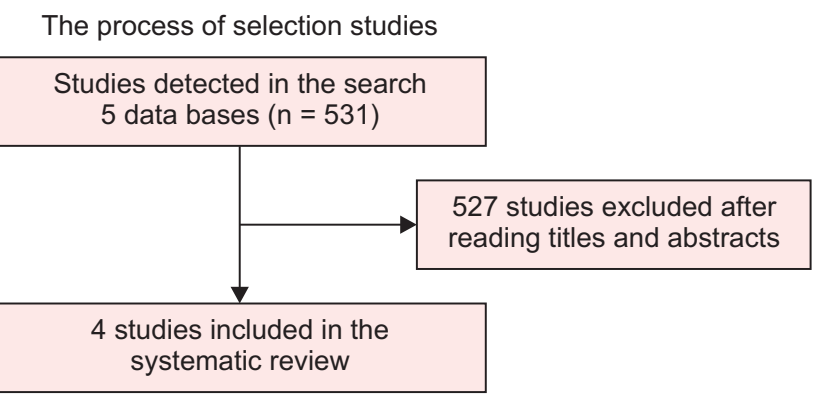

Fig. 1. The process of selection of studies to include into systematic review. 


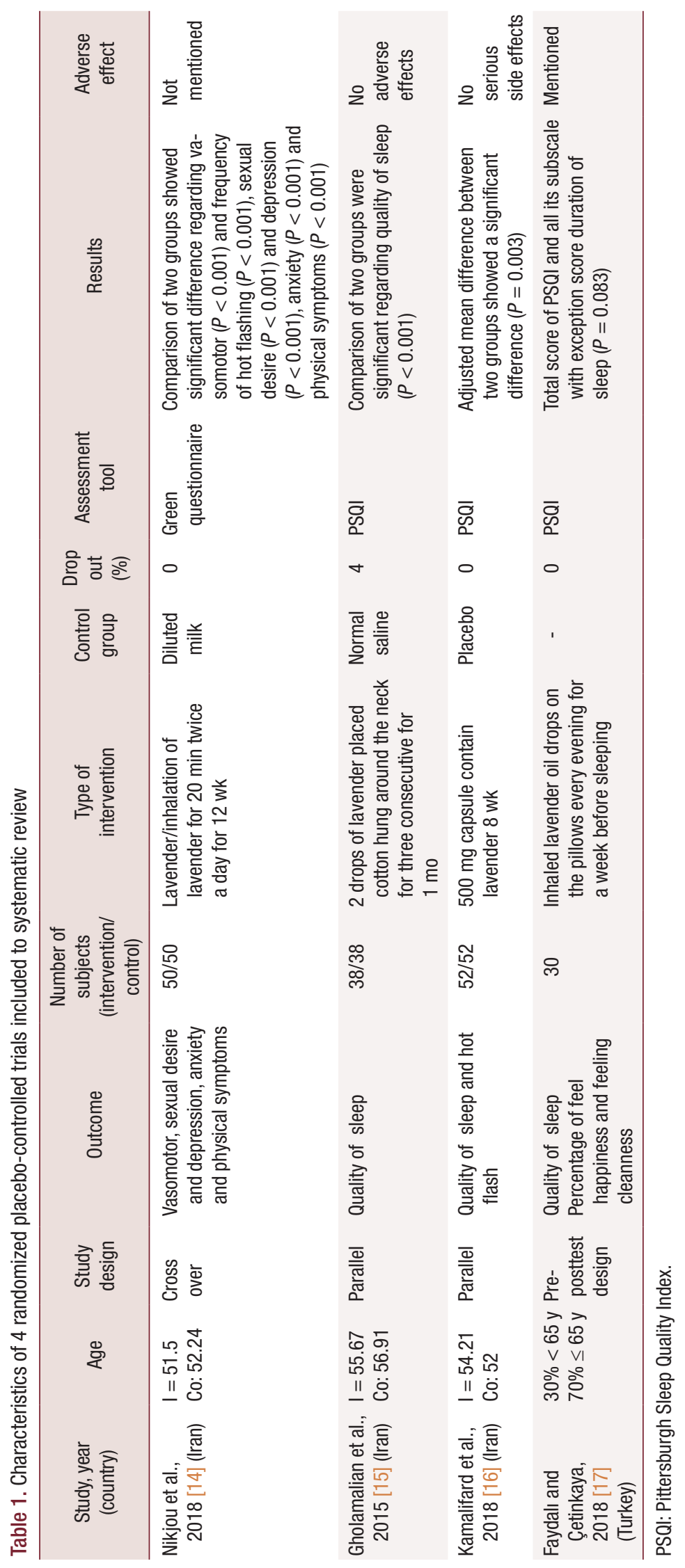


Table 2. The risk of bias assessment using Cochrane Collaboration's tool

\begin{tabular}{|c|c|c|c|c|}
\hline Variable & Kazemzadeh & Gholamalian & Nikjou & Faydalı \\
\hline Randomization & & & & Pretest-posttest design \\
\hline Mention randomization & * & * & * & \\
\hline Method: appropriate & * & * & * & \\
\hline Method: inappropriate & - & - & - & \\
\hline \multicolumn{5}{|l|}{ Blinding } \\
\hline Mention blinding & * & * & * & \\
\hline Method: appropriate & * & * & * & \\
\hline Method: inappropriate & - & - & - & \\
\hline \multicolumn{5}{|l|}{ Sample } \\
\hline Account of all patients & * & * & * & \\
\hline Intention to treat & No & No & No & \\
\hline Baseline comparability & Yes & Yes & No & \\
\hline
\end{tabular}

groups to receive aromatherapy with lavender and diluted milk as control. The comparison of treatment groups using Student $t$ test showed significant improvement in lavender group than placebo group $(P<0.001)$ regarding vasomotor symptoms.

\section{Quality of sleep}

Two trials assessed the effect of lavender on the quality of sleep. In first trial, Gholamalian et al. [15] evaluated the efficacy of aromatherapy with lavender compared to normal saline as control regarding quality of sleep on 76 postmenopausal women. The pre/post comparison of total Pittsburgh Sleep Quality Index score (PSQI) showed a statistically significant improvement in the lavender group $(P<0.001)$. However, total PSQI score was slightly worst in control group (normal saline). The comparison of two groups indicated significant difference $(P<0.001)$.

In second trial, Kamalifard et al. [16] in duplicate publication published in Persian databases randomized 104 patients to two groups to receive 500-mg lavender capsule $(n=52)$ or $500-m g$ placebo capsule $(n=52)$. Demographic characteristics of the menopausal subjects, such as the mean age and years since menopause were significantly different between groups. Adjusted mean difference between two groups showed a significant difference, with an improvement in total PSQI score in lavender group compared to placebo group $(P=0.003)$. In third study, Faydalı and Çetinkaya [17] conducted a pre- and posttest design to assess the effect of aromatherapy with lavender on the quality of sleep in elderly people. Total score PSQI $(P=0.001)$ and its subscales of sleep disturbance $(P=0.018)$, sleep latency $(P=0.002)$, day of dysfunction due to sleepiness $(P=$ $0.026)$, sleep efficiency $(P=0.018)$, overall sleep quality $(P=0.03)$, and need meds to sleep $(P=0.033)$ showed a significant improvement, except for duration of sleep $(P$ $=0.083$ ). Additionally, 93 patients believed that treatment with lavender was effective in promoting sleep quality, and $80.0 \%$ mentioned that they woke up rested in the morning.

\section{Sexual function}

Nikjou et al. [14] conducted a double-blinded crossover clinical trial to evaluate the efficacy of aromatherapy with lavender $(n=50)$ compared to placebo $(n=50)$ on the sexual desire among the menopausal with complaint of hot flash. The comparison of treatment groups using $t$-student test showed significant improvement in lavender than placebo group regarding sexual desire $(P<$ $0.001)$.

\section{Psychological symptoms}

In the first study, Nikjou et al. [14] conducted a double-blinded cross-over clinical trial. The menopausal women with complaint of hot flash were randomized into two equal groups to receive aromatherapy with lavender and diluted milk, as control. The comparison of two groups using Student $t$ test indicated a significant decrease in both anxiety $(P<0.001)$ and depression $(P$ $<0.001)$ in lavender groups compared to placebo. In seconded study, Faydalı and Çetinkaya [17] conducted 
a pre- and posttest design to assess the effect of aromatherapy with lavender on the quality of sleep in elderly people. Secondary outcome was feelings of relaxation, happiness and cleanness. $66.7 \%, 70.0 \%$, and $53.3 \%$ of subjects reported feelings of relaxation, happiness, and cleanness effects of lavender, respectively.

\section{Physical signs}

According to second study, Nikjou et al. [14] conducted a double-blinded cross-over clinical trial. Them menopausal women with complaint of hot flash were randomized into two equal groups to receive aromatherapy with lavender and diluted milk, as control. The comparison of treatment groups using t-student test showed significant improvement in lavender group than placebo group regarding physical signs $(P<0.001)$.

\section{DISCUSSION}

Menopause is a natural life cycle of all females that occurs following the estrogen deficiency, probably resulting in insomnia, irritability, and depression $[1,2]$. Nanomedicine can used in treatment of menopausal symptoms [2]. Lavender has beneficial effect on some menopausal symptoms. To the best of our knowledge, this is first systematic review aiming to assess the efficacy of lavender on quality of sleep, sexual desire, psychological and physical symptom among menopausal and elderly women. The results suggested the effectiveness of lavender on all above symptoms.

Lavender may have both direct and indirect effect on the quality of sleep. Indirect effect may be related to other properties of lavender as previous studies showed the efficacy of lavender for sexual function $[14,18]$, hot flash [4], physical [14], and psychological [14,19] symptoms.

The main compounds in lavender ( $L$. angustifolia) are linalool $(38.6 \%-76.9 \%)$, followed by lavandulol $(4.1 \%-8.6 \%)$, coumarin $(2.2 \%-21.8 \%)$, and terpinen4 -ol $(0.3 \%-14.3 \%)$ [20]. The lavender plays a sedative role similar to narcotic materials. There are several mechanisms of action for lavender to improve the sleep disorders, such as the acetylcholine secretion blocking and gamma-aminobutyric acid receptors interaction in the CNS [16]. The mechanism of action of lavender in alleviating hot flash can be attributed to a decrease in stress hormone and stimulation of beta-endorphin secretion [4].

The lavender was administrated in capsule form and aroma in this systematic review. Psychological and physiological functions of aromatherapy have been showed. The olfactory nerve cells activated by odors releasing from aromas transmitted signals to limbic system. Depending on the type of aroma, neuro messengers such as enkefaline, noradrenalin and serotonin are released by brain. These neuro messengers related with nervous and other boy system [21].

One of the main limitations of this study was small sample size. Future study should, therefore, be performed on larger sample size to achieve subgroup analysis. Almost all of studies compared the effect of lavender with placebo, so the comparison of the lavender effect is suggested with other conventional treatment. None of the studies assessed the intention to treat analysis. Future works should be designed and described based on consort guidelines. Confounding factors such as body mass index, stress level and diet may affect the sleep function $[15,16]$, which should be controlled in future investigations. Validity and reliability of PSQI were not reported in none of studies included in systematic review. It is impossible to consider the blinding method in aromatherapy; however, size, color and shape of control and treatment groups were identical in the included trials.

The present results suggested the effectiveness of lavender in capsule form or aromatherapy on the quality of sleep, sexual desire, depression, anxiety and physical symptom in among menopausal and elderly people. These results should be interpreted with caution in light of mentioned limitations. The well-designed studies are required with larger sample size to draw the definite conclusion.

\section{CONFLICT OF INTEREST}

No potential conflict of interest relevant to this article was reported.

\section{REFERENCES}

1. Kamalifard M, Farshbaf Khalili A, Namadian M, Herizchi S, Ranjbar Y. Comparison of the effect of lavender and bitter orange on depression in menopausal women: a triple-blind randomized controlled trial. IJWHR 2017; 5: 224-30.

2. Kargozar R, Azizi H, Salari R. A review of effective herbal medicines in controlling menopausal symptoms. Electron Physician 2017; 9: 5826-33.

3. Avis NE, Stellato R, Crawford S, Bromberger J, Ganz P, Cain V, et 
al. Is there a menopausal syndrome? Menopausal status and symptoms across racial/ethnic groups. Soc Sci Med 2001; 52: 345-56.

4. Kazemzadeh R, Nikjou R, Rostamnegad M, Norouzi H. Effect of lavender aromatherapy on menopause hot flushing: a crossover randomized clinical trial. J Chin Med Assoc 2016; 79: 489-92.

5. Geller SE, Studee L. Contemporary alternatives to plant estrogens for menopause. Maturitas 2006; 55 Suppl 1: S3-13.

6. Fattah A. Effect of phytoestrogen on depression and anxiety in menopausal women: a systematic review. J Menopausal Med 2017; 23: $160-5$.

7. Ghazanfarpour M, Amini E, Khadivzadeh T, Babakhanian M, Nouri B, Rakhshandeh $\mathrm{H}$, et al. The effect of short-term treatment with fennel on bone density in postmenopausal women: a randomized controlled trial. J Menopausal Med 2017; 23: 124-30.

8. Saghafi N, Ghazanfarpour M, Khadivzadeh T, Babakhanian M, Afiat M. The effect of Foeniculum vulgare (Fennel) on body composition in postmenopausal women with excess weight: a doubleblind randomized placebo-controlled trial. J Menopausal Med 2017; 23: 166-71.

9. Bakhtshirin F, Abedi S, YusefiZoj P, Razmjooee D. The effect of aromatherapy massage with lavender oil on severity of primary dysmenorrhea in Arsanjan students. Iran J Nurs Midwifery Res 2015; 20: 156-60.

10. Peana AT, Moretti MDL. Linalool in essential plant oils: pharmacological effects. In: Preedy VR, Watson RR, editors. Botanical medicine in clinical practice. Walingford: CAB International; 2008.

11. Fißler M, Quante A. A case series on the use of lavendula oil capsules in patients suffering from major depressive disorder and symptoms of psychomotor agitation, insomnia and anxiety. Complement Ther Med 2014; 22: 63-9.

12. Bakhsha F, Mazandarani M, Aryaei M, Jafari SY, Bayate H. Phytochemical and anti-oxidant activity of lavandula angustifolia mill. Essential oil on preoperative anxiety in patients undergoing diag- nostic curettage. Int J Women's Health Reprod Sci 2014; 2: 268-71.

13. Jadad AR, Moore RA, Carroll D, Jenkinson C, Reynolds DJ, Gavaghan DJ, et al. Assessing the quality of reports of randomized clinical trials: is blinding necessary? Control Clin Trials 1996; 17: 1-12.

14. Nikjou R, Kazemzadeh R, Asadzadeh F, Fathi R, Mostafazadeh F. The effect of lavender aromatherapy on the symptoms of menopause. J Natl Med Assoc 2018; 110: 265-9.

15. Gholamalian F, Tadayon M, Abedi P, Haghighizadeh MH. The effect of lavender aromatherapy on sleep quality in postmenopausal women. Iran J Obstet Gynecol Infertil 2015; 18: 18-25.

16. Kamalifard M, Farshbaf-Khalili A, Namadian M, Ranjbar Y, Herizchi S. Comparison of the effect of lavender and bitter orange on sleep quality in postmenopausal women: a triple-blind, randomized, controlled clinical trial. Women Health 2018; 58: 851-65.

17. Faydalı S, Çetinkaya F. The effect of aromatherapy on sleep quality of elderly people residing in a nursing home. Holist Nurs Pract 2018; 32: 8-16.

18. Malakouti J, Farshbaf Khalili A, Asghari Jafarabadi M, Jabbari F. Effect of combined inhaler aromatherapy on sexual function in postmenopausal women: a randomized controlled trial. Iran J Obstet Gynecol Infertil 2016; 19: 9-15.

19. Lotfipur-Rafsanjani SM, Vaziri-Nejad R, Ismailzadeh S, AnsariJaberi A, Bekhradi R, Ravari A, et al. Comparison of the efficacy of massage therapy and aromatherapy massage with geranium on depression in postmenopausal women: a clinical trial. Zahedan J Res Med Sci 2015; 17: 29-33.

20. Moussi Imane M, Houda F, Said Amal AH, Kaotar N, Mohammed T, Imane R, et al. Phytochemical composition and antibacterial activity of moroccan lavandula angustifolia mill. J Essent Oil Bear Plants 2017; 20: 1074-82.

21. Ali B, Al-Wabel NA, Shams S, Ahamad A, Khan SA, Anwar F. Essential oils used in aromatherapy: a systemic review. Asian Pac J Trop Biomed 2015; 5: 601-11. 